\title{
PEP1 regulates perennial flowering in Arabis alpina
}

\author{
Renhou Wang ${ }^{1}$, Sara Farrona ${ }^{1}$, Coral Vincent ${ }^{1}$, Anika Joecker ${ }^{1}$, Heiko Schoof ${ }^{1}$, Franziska Turck $^{1}$, \\ Carlos Alonso-Blanco ${ }^{2}$, George Coupland ${ }^{1} \&$ Maria C. Albani ${ }^{1}$
}

Annual plants complete their life cycle in one year and initiate flowering only once, whereas perennials live for many years and flower repeatedly. How perennials undergo repeated cycles of vegetative growth and flowering that are synchronized to the changing seasons has not been extensively studied ${ }^{1}$. Flowering is best understood in annual Arabidopsis thaliana ${ }^{2,3}$, but many closely related species, such as Arabis alpina ${ }^{4,5}$, are perennials. We identified the A. alpina mutant perpetual flowering 1 (pep1), and showed that PEP1 contributes to three perennial traits. It limits the duration of flowering, facilitating a return to vegetative development, prevents some branches from undergoing the floral transition allowing polycarpic growth habit, and confers a flowering response to winter temperatures that restricts flowering to spring. Here we show that PEP1 is the orthologue of the A. thaliana gene FLOWERING LOCUS C (FLC). The FLC transcription factor inhibits flowering until A. thaliana is exposed to winter temperatures ${ }^{6,7}$, which trigger chromatin modifications that stably repress $F L C$ transcription ${ }^{8-11}$. In contrast, $P E P 1$ is only transiently repressed by low temperatures, causing repeated seasonal cycles of repression and activation of PEP1 transcription that allow it to carry out functions characteristic of the cyclical life history of perennials. The patterns of chromatin modifications at FLC and PEP1 differ correlating with their distinct expression patterns. Thus we describe a critical mechanism by which flowering regulation differs between related perennial and annual species, and propose that differences in chromatin regulation contribute to this variation.

Perennial plants repeatedly cycle between vegetative and reproductive development. In temperate climates these cycles are synchronized to the changing seasons, for example, by restricting flowering to spring and summer ${ }^{12}$ or by arresting growth in the autumn ${ }^{13}$. Annual and perennial plants also show differences in the behaviour of shoot meristems-groups of undifferentiated cells from which all aerial tissues are derived. In annual plants all shoot meristems initiate reproductive development at similar times, a behaviour called monocarpy. In contrast, perennials are polycarpic and maintain vegetative growth after flowering, which allows them to flower and set seed many times during their lifetime. Vegetative growth is maintained either by conserving some meristems in the vegetative state after flower initiation ${ }^{14}$ or by reverting back to vegetative development after flowering ${ }^{15}$.

Control of the floral transition is best understood in the annual monocarpic model species Arabidopsis thaliana ${ }^{2,3}$, a member of the Brassicaceae. Many other Brassicaceae species are polycarpic perennials, and therefore the molecular mechanisms underlying the difference between monocarpic and polycarpic plants can be approached by comparing $A$. thaliana with its close relatives. Similar comparative approaches were recently used to study the development of compound leaves ${ }^{16}$ and to analyse the basis of heavy metal tolerance ${ }^{17}$. The arcticalpine perennial Arabis alpina is a member of the Brassicaceae $e^{4,5}$ that has favourable characteristics for use as a model perennial species, such as being diploid and self-fertile, with a relatively small genome, and being susceptible to transformation by Agrobacterium tumefaciens. Here we describe a molecular-genetic analysis of the perennial traits seasonal flowering and polycarpy in A. alpina. We demonstrate that the A. alpina gene PERPETUAL FLOWERING 1 (PEP1) encodes a MADS-box transcription factor that mechanistically links polycarpy and seasonal flowering. PEP1 is the orthologue of the $A$. thaliana gene FLOWERING LOCUS $C$ (FLC) and it is differently regulated in A. alpina, allowing the repeated response to seasonal cycles observed in perennial plants. This distinct pattern of regulation is related to species-specific differences in histone modifications at PEP1. These data provide insight into the mechanisms underlying evolution of life history in plants.

To facilitate a genetic study of flowering in A. alpina we first characterized the perennial growth habit and responses to environmental signals. The accession Pajares did not flower when grown continuously under long days (16 h light) or exposed to short days (8 h light) (Fig. 1a, Supplementary Fig. 1a and Methods). However, these plants flowered when exposed to low temperatures for several weeks (Fig. 1b), a treatment called vernalization. Although the flowering response to vernalization was independent of day length (Supplementary Fig. 1a), it was influenced by the duration of exposure to cold. Twelve weeks vernalization was sufficient to saturate the flowering response, causing all plants to produce fully developed inflorescences, whereas vernalization for shorter periods resulted in inflorescences that seemed to revert to vegetative growth (Fig. 1b, e and Supplementary Fig. 1b). Therefore, Arabis alpina Pajares plants only flower if exposed to prolonged vernalization treatments, and this response is independent of day length.

To understand the polycarpic behaviour of A. alpina, the fates of apical meristems of the main shoot and axillary shoots (branches) were followed before and after vernalization. The apices of the main shoot and axillary shoots formed before cold treatment produced flower buds during vernalization (Fig. 1c and Supplementary Figs 2 and $3 a-d)$. Mature flowers emerged when plants were subsequently moved back to normal growth temperatures. Vegetative shoots developed from meristems that were not present or were still at an early developmental stage at the onset of vernalization, contributing to the polycarpic growth habit of the plant (Supplementary Fig. 3e, f). These shoots continued growing vegetatively until plants were again exposed to vernalization, inducing another round of flowering (Fig. 1d-h). Therefore, perenniality in this species involves maintenance of vegetative development after flowering, and the requirement for vernalization to induce flowering during each annual cycle.

To study the molecular mechanisms controlling flowering of A. alpina, mutants showing an impaired vernalization response were identified. From a total of $25,000 \mathrm{M}_{2}$ seedlings, two mutants were isolated that lacked obligate vernalization requirement. The most extreme early flowering mutant was called perpetual flowering 1 (pep1). This mutant flowered with approximately 25 leaves under long 

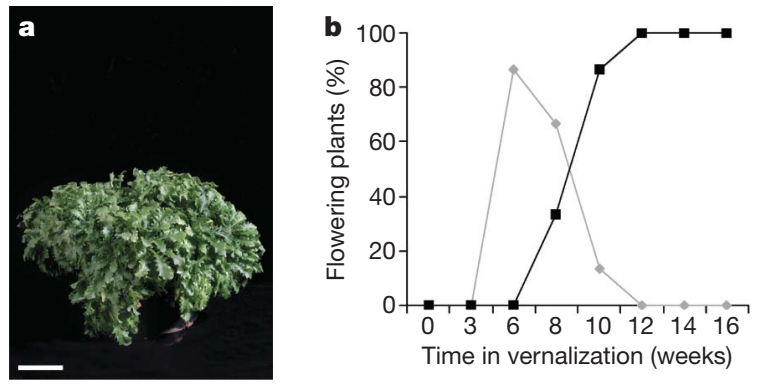

c
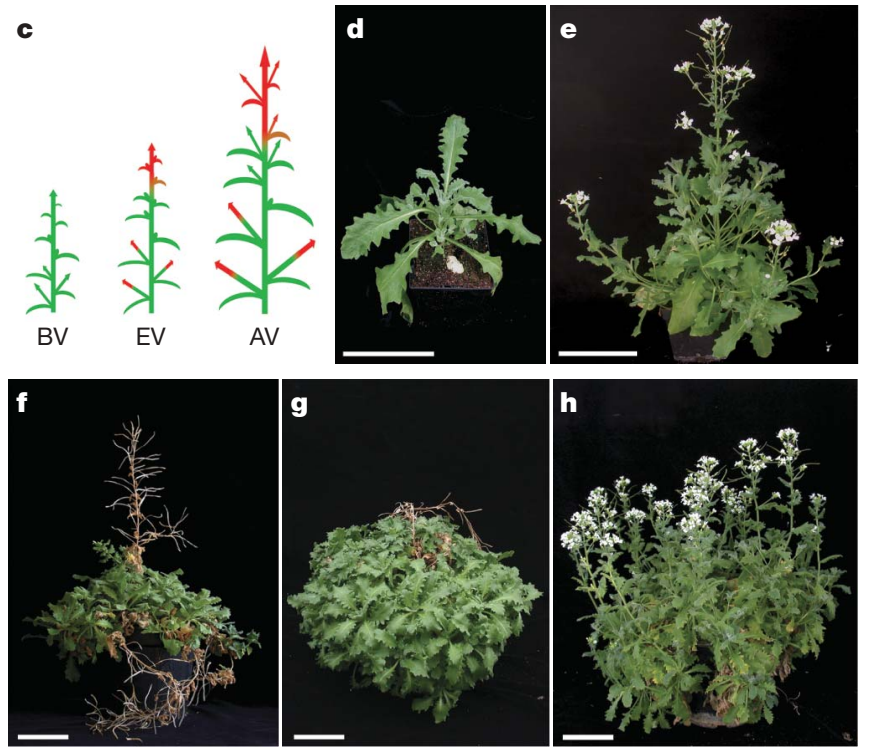

Figure 1 Arabis alpina is a polycarpic perennial that requires vernalization each year to flower. a, An A. alpina (accession Pajares) plant grown for 3 and a half years without vernalization. The plant has not flowered.

b, Quantification of the duration of vernalization required for flowering of the Pajares accession. Plants were grown for 8 weeks in long days, vernalized for different times and subsequently grown in long days. Shown in grey are plants that responded partially to vernalization by producing a few flowers but also producing vegetative characteristics on the inflorescence (see also Supplementary Fig. 1b). Shown in black are plants that completely responded to vernalization and produced fully developed inflorescences. Twelve plants were scored at each time point. c, Diagram showing differential response of shoots to vernalization in A. alpina. The main shoot and axillary shoots are vegetative (green) before vernalization and become reproductive (red) by the end of the vernalization treatment. Axillary shoots that arise during or after vernalization remain vegetative (green). BV, before vernalization, plants grown for 8 weeks in long days; EV, end of a 12-week vernalization treatment under short days at $4{ }^{\circ} \mathrm{C} ; \mathrm{AV}$, after vernalization, plants grown under long days. d-h, Arabis alpina does not die after flowering. The reproductive part of each shoot sets seed and senesces whereas vegetative shoots maintain vegetative growth indefinitely until exposed to another round of vernalization. Pictures from the same A. alpina plant were taken at successive stages of its life cycle: after 8 weeks in long days (d), vernalized for 12 weeks and then transferred to long days for 3 weeks (e), after 14 weeks in long days (f), after 25 weeks in long days (g), and after a new round of 12 weeks vernalization and then again for 4 weeks in long days (h). Scale bars, $10 \mathrm{~cm}$.

days without exposure to vernalization, whereas wild-type plants never flowered under these conditions (Fig. 2a, b). In addition, the pep1 mutant flowered continuously for at least 12 months (Supplementary Fig. 4a). To compare the duration of the flowering season of the pep1 mutant and wild-type plants, both genotypes were vernalized and the duration of flowering was measured. Reproductive shoots in wild-type plants completed flowering 14 weeks after return to warm temperatures under long days, whereas under the same conditions pep1 plants flowered for a further 5 months until the experiment was terminated (Fig. 2c, d). Flowering of wild-type plants was restricted to apical meristems of shoots that were present before vernalization, whereas the pep1 mutant continued flowering from
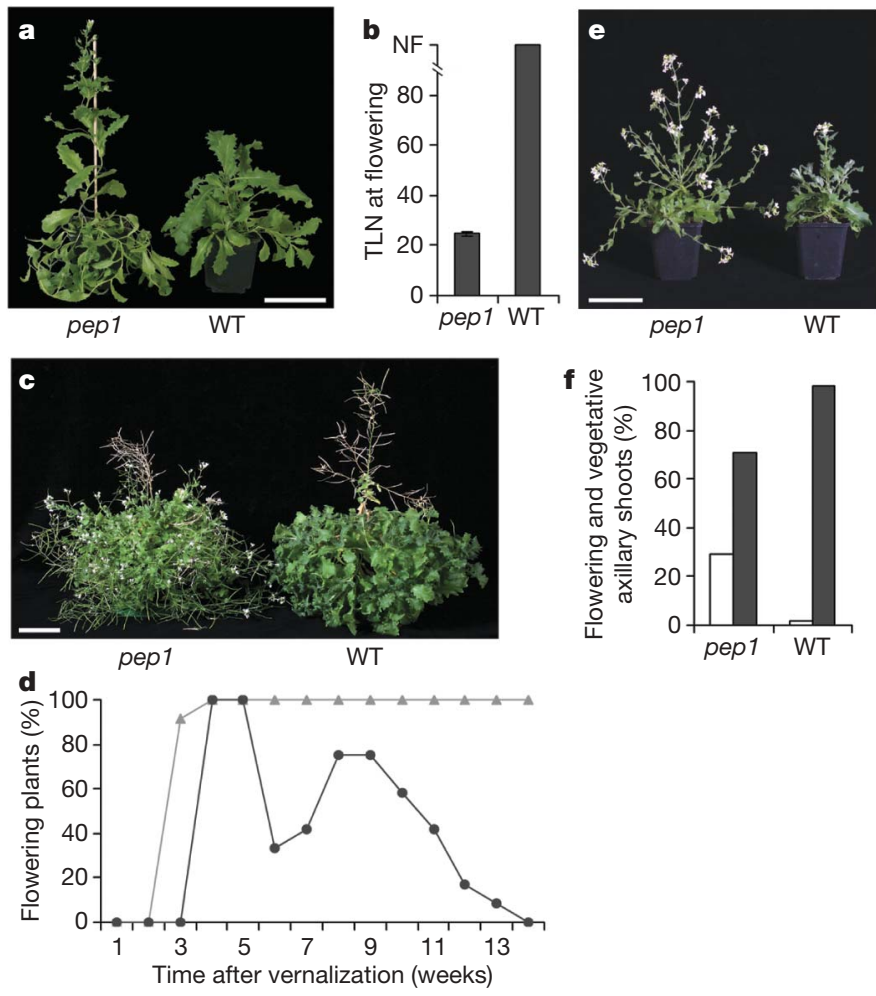

Figure 2 | PEP1 restricts the flowering phase and enhances polycarpy of A. alpina. a, The pep1 mutant flowers without vernalization. b, Flowering time of pep1 plants compared to wild type (WT) without vernalization. NF, no flowering; TLN, total leaf number. c, pep1 mutants show longer periods of flowering than wild-type plants. Plants were grown for 14 weeks under long days after 12 weeks vernalization. $d$, Duration of the flowering phase of pep 1 (grey) and wild-type plants (dark grey) after vernalization. e, More axillary branches undergo flowering in pep1 plants than in wild-type as shown by plants grown for 3 weeks under long days after 12 weeks vernalization. f, Percentage of vegetative and flowering axillary shoots produced by pep 1 and wild-type plants. Plants were scored when the first open flower was present. Flowering shoots (white) and vegetative shoots (dark grey) are shown. In c-f, plants were grown under long days for 5 weeks before being vernalized for 12 weeks. $n=12(\mathrm{~b}, \mathrm{~d}, \mathrm{f})$; scale bars, $10 \mathrm{~cm}$.

secondary axillary shoots (Supplementary Fig. 4b). Moreover, the pep1 mutant showed altered polycarpic behaviour. More shoots were induced to flower in the pep1 mutant because axillary shoots that emerged during or after vernalization flowered in the pep1 mutant but not in the wild-type plants (Fig. 2e, f and Supplementary Fig. 4c, d). Our data demonstrate that PEP1 acts at different stages in the perennial life cycle. PEP1 prevents flowering before vernalization, whereas after vernalization it acts to restrict the duration of flowering and contributes to polycarpy by blocking flowering of axillary shoots. These three functions all involve the repression of the floral transition and are likely to be mechanistically related.

The reduced requirement for vernalization in the pep1 mutant suggested that PEP1 may be an orthologue of an A. thaliana gene conferring a vernalization requirement. To test this idea we isolated the A. alpina orthologue of FLOWERING LOCUS C (FLC), which encodes a MADS-box transcription factor that has an important role in establishing vernalization requirement in $A$. thaliana $a^{6,7}$ and probably other annual Brassicaceae species ${ }^{18,19}$. AaFLC is present in a genomic region showing microsynteny with the $A$. thaliana chromosomal region containing FLC (Fig. $3 a$ and Supplementary Table 1), and encodes a protein more closely related to FLC than to any other A. thaliana protein (Supplementary Fig. 5a). The function of AaFLC was tested by expressing the AaFLC complementary DNA from the CaMV35S promoter in the accession Columbia-0, which expresses the endogenous FLC gene at low levels ${ }^{6,7}$. The transgenic plants were late 
a
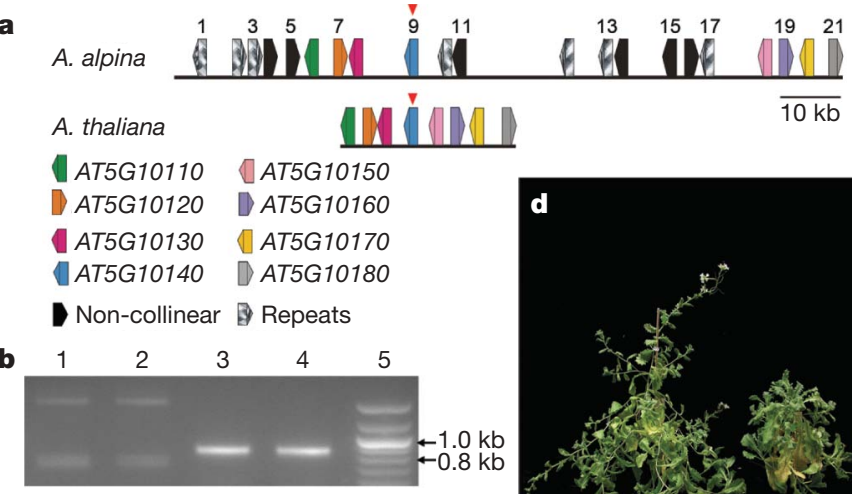

c WT ...AAATTGTTGAAAG GTTAGTATTGGTTA... pep1 ....AAATTGTTGAAAG ATTAGTATTGGTTA... Exon Intron

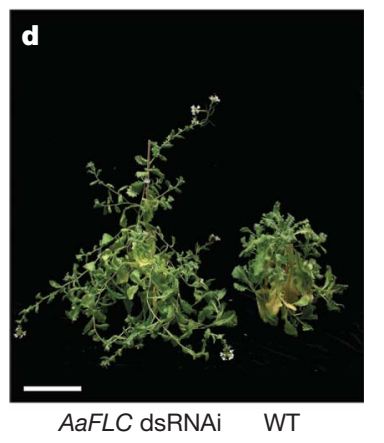

Figure $3 \mid$ PEP1 is the Arabis alpina orthologue of A. thaliana FLC. a, Conserved synteny between the Arabis alpina (top panel) and A. thaliana (bottom panel) chromosomal regions containing AaFLC and Arabidopsis $F L C$, respectively. Vertical arrowheads show AaFLC and Arabidopsis FLC in each panel. Horizontal arrowheads illustrate genes and their orientation. Coloured arrowheads show syntenic genes, whereas black and striped arrowheads show the non-collinear genes and repeats, respectively. Genes (1-21) on the BAC containing the AaFLC gene are annotated in Supplementary Table 1. GenBank accession number FJ543377. b, Accumulation of the full-length $A a F L C$ transcript is impaired in pep1 mutant plants. Amplified products from cDNA derived from pep1 apices (lane 1), pep1 leaves (lane 2), wild-type apices (lane 3 ) and wild-type leaves (lane 4). Lane 5 contains a 100-base-pair (bp) standard (NEB). Primers were designed to amplify by PCR the full-length coding sequence of AaFLC (819bp). AaFLC cDNA in the pep1 mutant and wild-type plant were sequenced and the detected splice variants are shown in Supplementary Fig. 6. In the pep1 mutant, splice variants that were larger and smaller than the fully spliced $A a F L C O R F$ were detected, accounting for the differently sized cDNAs detected in lanes 1 and 2. c, Mutation in the AaFLC gene in the pep1 mutant. The G-to-A mutation at the splice donor site of exon 3 is indicated in green (wild type, WT) and red (pep1). d, Transgenic 35S:AaFLC doublestranded (ds)RNAi A. alpina Pajares plants flower without vernalization. See also Supplementary Figs 5 and 8 . Scale bar, $10 \mathrm{~cm}$.

flowering (Supplementary Fig. 5b, c), demonstrating that AaFLC can act as a floral repressor in A. thaliana as previously shown for $\mathrm{FLC}^{6,7}$.

To test the relationship between AaFLC and PEP1, the expression of AaFLC messenger RNA was compared in pep1 and wild-type plants. Full-length AaFLC mRNA was below the level of detection in the pep1 mutant (Fig. 3b and Supplementary Fig. 6), and by sequencing the $A a F L C$ genomic region a mutation (G-to-A) was identified at the $5^{\prime}$ splice junction of the third intron (Fig. 3c). In the pep1 mutant AaFLC mRNAs of different sizes were detected (Fig. 3b), and therefore AaFLC cDNAs made from pep1 RNA were sequenced. None of the $14 \mathrm{cDNAs}$ tested contained the full AaFLC open reading frame (ORF; Supplementary Fig. 6). In contrast, the cDNA containing the full ORF was by far the most abundant form detected in wild-type $A$. alpina plants (Fig. 3b and Supplementary Fig. 6). These data support the idea that the mutation present in AaFLC in the pep1 mutant impairs production of the properly spliced mRNA.

We further tested whether reduced AaFLC activity was the cause of the pep1 mutant phenotype. First, genetic linkage between pep1 and the mutation in $A a F L C$ was tested in an $\mathrm{F}_{2}$ population constructed by backcrossing the pep1 mutant to wild type. Under continuous long days, the ratio of flowering to non-flowering $\mathrm{F}_{2}$ plants was approximately 1:3 (Supplementary Fig. 7). Genotypic data demonstrated that all of the flowering plants were homozygous for the mutation in $A a F L C$, and that all non-flowering plants were either heterozygous or homozygous for the wild-type allele (Supplementary Fig. 7). This experiment demonstrated that pep1 is genetically linked to AaFLC. Second, transgenic plants, in which the level of AaFLC mRNA was reduced by RNA interference (RNAi), were generated. These plants showed the characteristic phenotypes of pep1 mutant plants. They flowered without vernalization, showed a longer duration of flowering and produced more flowering branches compared to the wild type (Fig. 3d and Supplementary Figs 5d, e and 8). These experiments further indicated that the pep1 mutant phenotype is caused by impaired AaFLC activity and therefore AaFLC will be referred to as PEP1.

In A. thaliana, FLC transcription is repressed during vernalization, and after return to normal growth temperatures FLC repression is maintained $^{6-8,10}$. The behaviour of pep1 mutants suggested that PEP1 functions before and after vernalization. To determine the PEP1 expression pattern in A. alpina, apices and leaves of wild-type plants were analysed before, during and after vernalization using quantitative PCR with reverse transcription (qRT-PCR). During vernalization, PEP1 transcript levels decreased markedly in shoot apices and leaves, and reached much lower levels at the end of the low temperature treatment, as observed in A. thaliana. However, after return to normal growth temperatures, PEP1 mRNA levels increased in all tissues tested (Fig. 4a, b). To determine the spatial expression patterns of PEP1, RNA in situ hybridization experiments were performed. Before vernalization, PEP1 mRNA was strongly detected in the shoot apical meristem, the adjacent axillary meristems and young leaves (Fig. 4c). At the end of vernalization, PEP1 mRNA could not be detected in the flower buds at the shoot apical meristem, and was only weakly expressed in the primordia of newly formed vegetative axillary shoots (Fig. 4d, f). In agreement with the qRT-PCR results, PEP1 transcript levels were restored after vernalization in both flowering and vegetative meristems (Fig. 4e, g). Therefore, in contrast to FLC in A. thaliana, after vernalization PEP1 mRNA is restored to similar levels to those present before vernalization.

In $A$. thaliana stable repression of FLC by vernalization correlates with modification of histone $\mathrm{H} 3$ ( refs 8,9 ), and particularly with the addition of three methyl groups to the lysine residue at position 27 $(\mathrm{H} 3 \mathrm{~K} 27 \mathrm{me} 3)^{11,20-22}$. During vernalization the level of this histone modification increases close to the transcription start site of FLC, and in growing tissues it persists after return to normal growth temperatures, spreading across the gene and correlating with stable transcriptional repression ${ }^{11}$. In A. alpina the level of H3K27me3 also increased at PEP1 during vernalization, but its levels decreased again after vernalization (Fig. 4h). Therefore, in contrast to FLC in A. thaliana, in A. alpina the H3K27me3 mark accumulated at PEP1 during vernalization but did not persist after vernalization.

Thus, temporal changes in PEP1 expression contribute to the perennial life history of A. alpina. Repression of PEP1 transcription during vernalization and reactivation after return to warm temperatures correlate with unstable modifications of $\mathrm{H} 3 \mathrm{~K} 27 \mathrm{me} 3$ at the PEP1 locus. This observation is in contrast to FLC expression patterns in growing tissues of A. thaliana, in which FLC expression is stably repressed by vernalization and the $\mathrm{H} 3 \mathrm{~K} 27 \mathrm{me} 3$ chromatin mark persists at the locus after vernalization ${ }^{10,11,20-22}$. Annual plants such as A. thaliana only flower once in their lifetime, and therefore to maximise seed production all growing shoot meristems undergo the transition to flowering when the environmental conditions are optimal. Thus, stable repression of the floral repressor FLC by vernalization is consistent with the monocarpic life strategy of A. thaliana. Mutations in a component of the polycomb-group protein complex required to produce the $\mathrm{H} 3 \mathrm{~K} 27 \mathrm{me} 3$ mark prevent stable repression of FLC by vernalization in Arabidopsis, indicating that this mark is required for epigenetic silencing of $F L C^{10,20}$. Moreover, Arabidopsis accessions that show variation in the duration of vernalization required to saturate the flowering response show differences in the accumulation of the H3K27me3 mark at the FLC locus during vernalization ${ }^{23}$. However, a reduction in the level of the mark after vernalization, as we observed in apices of fully vernalized $A$. alpina plants, has not been observed in growing tissues of $A$. thaliana, indicating that this aspect of FLC/PEP1 regulation differs between species. Nevertheless, in A. thaliana the H3K27me3 mark at FLC does not persist in cells of fully expanded leaves that are 


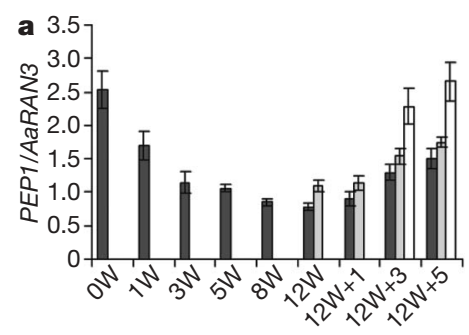

b 4.0
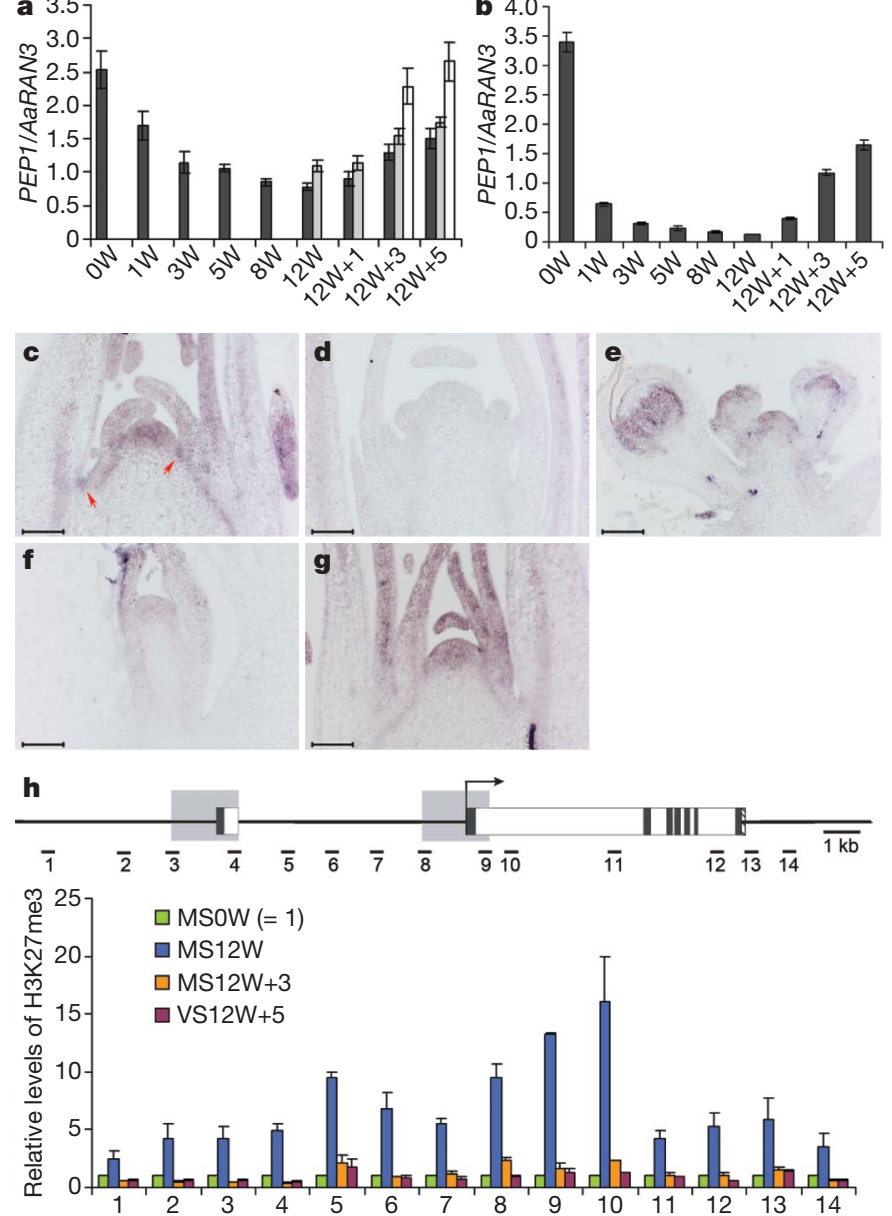

Figure 4 | Repression of PEP1 expression by vernalization is unstable and correlated with changes in histone methylation. a, b, PEP1 mRNA levels fall in apices (a) and leaves (b) during vernalization treatment (1 to 12 weeks $(\mathrm{W}))$, and are restored after vernalization $(12 \mathrm{~W}+1$ to 5 weeks). RNA levels were measured by qRT-PCR. In a, dark grey bars represent main-shoot apices. Grey bars represent apices from axillary shoots produced before vernalization. PEP1 expression in these axillary shoots was only measured at $12 \mathrm{~W}, 12 \mathrm{~W}+1,12 \mathrm{~W}+3$ and $12 \mathrm{~W}+5$ time points. White bars represent apices from axillary shoots produced after vernalization. In $\mathbf{a}$ and $\mathbf{b}$ the means of three technical replicates are shown; error bars indicate s.d. c-e, In situ hybridization of PEP1 mRNA in the main-shoot apex after 8 weeks growth in long days before vernalization (c), followed by 12 weeks vernalization (d), and after a further 4 weeks in long days (e). Arrows highlight axillary meristems that will develop into vegetative axillary shoots during or after vernalization. $\mathbf{f}, \mathbf{g}$, In situ hybridization of PEP1 mRNA in vegetative axillary shoots at the end of 12 -weeks vernalization (f) and after a further 4 weeks in long days (g). Scale bars, $100 \mathrm{~nm}$. h, The AaFLC locus showing the regions amplified by PCR after ChIP (top). Boxes represent exons (dark grey), introns (white), 3' untranslated region (UTR; striped) and a 2-kb duplicated region present at the locus (grey). H3K27me3 levels determined by ChIP of 14 regions on the AaFLC locus (bottom). All data points were divided by the H3K27me3 level before vernalization (8 weeks in long days) to provide relative values. The level before vernalization is therefore arbitrarily assigned a value of 1.0. Main-shoot apices of A. alpina plants were grown for 8 weeks in long days (MSOW), after 12 weeks vernalization (MS12W), and followed by 3 weeks in long days (MS12W +3 ). Apices from vegetative axillary shoots were grown for 5 weeks in long days after vernalization $(\mathrm{VS} 12 \mathrm{~W}+5)$. The data presented are the means of two technical replicates, error bars denote s.e.m. Similar patterns of modification were obtained in a second set of biological replicates.

no longer undergoing mitosis, indicating that in these tissues the mechanism required for persistence of this mark at FLC is not active ${ }^{11}$.

The instability of PEP1 repression in A. alpina allows PEP1 to block flowering of all meristems that did not undergo flowering during the preceding vernalization treatment, and therefore to take on roles in the perennial life cycle that are not required in the annual life cycle of A. thaliana. However, although differential regulation of PEP1 enhances perennial characters and confers seasonality, pep1 mutants are still long-lived and behave as perennial plants. This indicates that the differential regulation of PEP1 and FLC is not the only difference between $A$. thaliana and A. alpina that is responsible for perennialism. Furthermore, although our data demonstrate an important role for PEP1 as an inhibitor of flowering in the perennial cycle, the presence of extra components, including floral promoters that increase in expression before each flowering cycle, cannot be excluded. Nevertheless, our analysis of $P E P 1$ provides insight into the different mechanisms underlying seasonal flowering in perennial and annual plants consistent with their life history, and how gene functions involved in the control of seasonal flowering can be incorporated into perennial-specific traits such as polycarpy. The distinction between annual and perennial life histories has arisen independently many times in the flowering plants $^{24,25}$, and in the Brassicaceae both life histories occur in several genera $^{26}$, suggesting that they diversified independently. Furthermore, not all perennials flower in response to vernalization and FLC is not a universal regulator of vernalization requirement ${ }^{27}$, supporting the idea that different mechanisms must regulate the perennial growth habit in other groups of plants. Species-specific traits can evolve through alterations in the expression patterns of regulatory genes ${ }^{28}$. The differences in histone modifications at FLC and PEP1 in A. thaliana and A. alpina, respectively, suggest that differences in chromatin regulation may be one of the mechanisms by which these alterations in gene expression patterns occur, thereby allowing diversification of rapidly evolving traits such as life history characters.

\section{METHODS SUMMARY}

Plant material. Arabis alpina L. accession Pajares was collected in the Cordillera Cantábrica mountain system in Spain $\left(42^{\circ} 59^{\prime} 32^{\prime \prime} \mathrm{N}, 5^{\circ} 45^{\prime} 32^{\prime \prime} \mathrm{W}\right.$; $1,400 \mathrm{~m}$ altitude) and self-fertilized for six generations by single-seed descent. Arabidopsis thaliana experiments were performed using the Columbia-0 accession.

Ethyl methanesulphonate mutagenesis. Arabis alpina seeds were mutagenized with $0.35 \%$ ethyl methanesulphonate (Sigma) for $8-9 \mathrm{~h}$. Twenty-five-thousand $\mathrm{M}_{2}$ seedlings (2,500 $\mathrm{M}_{1}$ families) were screened in long days at a $20^{\circ} \mathrm{C}$ controlled-environment glasshouse.

BAC analysis and sequencing. Two positive BAC clones were identified by screening an A. alpina BAC library made from accession Pajares (R. Castillo, unpublished data) using A. thaliana FLC as a probe without the sequence encoding the MADS box. The DNA sequence of a positively hybridizing BAC was determined using the Sanger method, assembled and annotated manually.

Plant transformation. Plasmid constructs were generated by cloning PCR-amplified fragments into GATEWAY compatible binary vectors and introduced into Agrobacterium strain GV3101 (pMP90RK). Transgenic A. thaliana and A. alpina were generated by the floral-dip method ${ }^{29}$.

Gene expression analysis. Total RNA was extracted using the RNeasy Plant Mini Kit (Qiagen) from expanded leaves, main-shoot apices and axillary-shoot apices grown before or after vernalization. cDNA synthesis and qRT-PCR were performed as previously described ${ }^{30}$.

In situ hybridization. In situ hybridization was performed on apices of the main shoot and of axillary shoots, as described previously ${ }^{30}$.

Chromatin immunoprecipitation. Chromatin immunoprecipitation (ChIP) was carried out using apices of the main shoots (MS) of A. alpina plants grown for 8 weeks in long days (MS0W), then vernalized for 12 weeks (MS12W), and finally grown for 3 weeks in long days (MS12W +3 ). Apices of axillary vegetative shoots grown during and after vernalization were collected after 5 weeks in long days (VS12W +5). Chromatin samples were immunoprecipitated with antiH3K27me3 (Upstate). Results were presented as a ratio of vernalized/non-vernalized samples and are the mean of two technical replicates.

Full Methods and any associated references are available in the online version of the paper at www.nature.com/nature.

Received 9 January; accepted 12 March 2009.

Published online 15 April 2009.

1. Battey, N. H. \& Tooke, F. Molecular control and variation in the floral transition. Curr. Opin. Plant Biol. 5, 62-68 (2002). 
2. Turck, F., Fornara, F. \& Coupland, G. Regulation and identity of florigen: FLOWERING LOCUS T moves center stage. Annu. Rev. Plant Biol. 59, 573-594 (2008).

3. Baurle, I. \& Dean, C. The timing of developmental transitions in plants. Cell 125, 655-664 (2006).

4. Ansell, S. W., Grundmann, M., Russell, S. J., Schneider, H. \& Vogel, J. C. Genetic discontinuity, breeding-system change and population history of Arabis alpina in the Italian Peninsula and adjacent Alps. Mol. Ecol. 17, 2245-2257 (2008).

5. Koch, M. A. et al. Three times out of Asia Minor: the phylogeography of Arabis alpina L. (Brassicaceae). Mol. Ecol. 15, 825-839 (2006).

6. Michaels, S. D. \& Amasino, R. M. FLOWERING LOCUS C encodes a novel MADS domain protein that acts as a repressor of flowering. Plant Cell 11, 949-956 (1999).

7. Sheldon, C. C. et al. The FLF MADS box gene: A repressor of flowering in Arabidopsis regulated by vernalization and methylation. Plant Cell 11, 445-458 (1999).

8. Bastow, R. et al. Vernalization requires epigenetic silencing of $F L C$ by histone methylation. Nature 427, 164-167 (2004).

9. Sung, S. \& Amasino, R. M. Vernalization in Arabidopsis thaliana is mediated by the PHD finger protein VIN3. Nature 427, 159-164 (2004)

10. Gendall, A. R., Levy, Y. Y., Wilson, A. \& Dean, C. The VERNALIZATION 2 gene mediates the epigenetic regulation of vernalization in Arabidopsis. Cell 107, 525-535 (2001).

11. Finnegan, E. J. \& Dennis, E. S. Vernalization-induced trimethylation of histone $\mathrm{H} 3$ lysine 27 at FLC is not maintained in mitotically quiescent cells. Curr. Biol. 17, 1978-1983 (2007).

12. Battey, N. H. Aspects of seasonality. J. Exp. Bot. 51, 1769-1780 (2000).

13. Bohlenius, $\mathrm{H}$. et al. CO/FT regulatory module controls timing of flowering and seasonal growth cessation in trees. Science 312, 1040-1043 (2006).

14. Foster, T., Johnston, R. \& Seleznyova, A. A morphological and quantitative characterization of early floral development in apple (Malus $\times$ domestica Borkh.). Ann. Bot. (Lond.) 92, 199-206 (2003).

15. Diomaiuto, J. Periodic flowering or continual flowering as a function of temperature in a perennial species: the Ravenelle wallflower (Cheiranthus cheiri L.). Phytomorphology 38, 163-171 (1988).

16. Hay, A. \& Tsiantis, M. The genetic basis for differences in leaf form between Arabidopsis thaliana and its wild relative Cardamine hirsuta. Nature Genet. 38, 942-947 (2006).

17. Hanikenne, M. et al. Evolution of metal hyperaccumulation required cis-regulatory changes and triplication of HMA4. Nature 453, 391-395 (2008).

18. Schranz, M. E. et al. Characterization and effects of the replicated flowering time gene FLC in Brassica rapa. Genetics 162, 1457-1468 (2002)
19. D'Aloia, M., Tocquin, P. \& Perilleux, C. Vernalization-induced repression of FLOWERING LOCUS C stimulates flowering in Sinapis alba and enhances plant responsiveness to photoperiod. New Phytol. 178, 755-765 (2008).

20. Schubert, D. et al. Silencing by plant Polycomb-group genes requires dispersed trimethylation of histone $\mathrm{H} 3$ at lysine 27. EMBO J. 25, 4638-4649 (2006).

21. Sung, S., Schmitz, R. J. \& Amasino, R. M. A. PHD finger protein involved in both the vernalization and photoperiod pathways in Arabidopsis. Genes Dev. 20, 3244-3248 (2006).

22. Greb, T. et al. The PHD finger protein VRN5 functions in the epigenetic silencing of Arabidopsis FLC. Curr. Biol. 17, 73-78 (2007).

23. Shindo, C., Lister, C., Crevillen, P., Nordborg, M. \& Dean, C. Variation in the epigenetic silencing of $F L C$ contributes to natural variation in Arabidopsis vernalization response. Genes Dev. 20, 3079-3083 (2006).

24. Thomas, H., Thomas, H. M. \& Ougham, H. Annuality, perenniality and cell death. J. Exp. Bot. 51, 1781-1788 (2000).

25. Bena, G., Lejeune, B., Prosperi, J.-M. \& Olivieri, I. Molecular phylogenetic approach for studying life-history evolution: the ambiguous example of the genus Medicago L. Proc. R. Soc. Lond. B 265, 1141-1151 (1998).

26. Beilstein, M. A., Al-Shehbaz, I. A. \& Kellogg, E. A. Brassicaceae phylogeny and trichome evolution. Am. J. Bot. 93, 607-619 (2006).

27. Yan, L. et al. The wheat VRN2 gene is a flowering repressor down-regulated by vernalization. Science 303, 1640-1644 (2004).

28. Prud'homme, B., Gompel, N. \& Carroll, S. B. Emerging principles of regulatory evolution. Proc. Natl Acad. Sci. USA 104, 8605-8612 (2007).

29. Clough, S. J. \& Bent, A. F. Floral dip: a simplified method for Agrobacteriummediated transformation of Arabidopsis thaliana. Plant J. 16, 735-743 (1998)

30. Searle, I. et al. The transcription factor FLC confers a flowering response to vernalization by repressing meristem competence and systemic signaling in Arabidopsis. Genes Dev. 20, 898-912 (2006).

Supplementary Information is linked to the online version of the paper at www.nature.com/nature.

Acknowledgements The authors would like to thank $P$. Sharma for growing plants and R. Bruggmann for running the gene prediction pipeline. The laboratories of H.S. and G.C. are partly funded by a core grant from the Max Planck Society.

Author Information The GenBank accession number for the PEP1 BAC sequence is FJ543377, and for the PEP1 CDNA sequence is FJ755930. Reprints and permissions information is available at www.nature.com/reprints. Correspondence and requests for materials should be addressed to G.C.

(coupland@mpiz-koeln.mpg.de) or M.C.A. (albani@mpiz-koeln.mpg.de). 


\section{METHODS}

Growth conditions and phenotypic characterization. Plants were grown in long-day growth cabinets $\left(16 \mathrm{~h}\right.$ light at $22^{\circ} \mathrm{C}$, and $8 \mathrm{~h}$ dark at $\left.18^{\circ} \mathrm{C}\right)$ or longday climate-controlled glasshouses $\left(16 \mathrm{~h}\right.$ light at $\left.20^{\circ} \mathrm{C}\right)$, short-day cabinets $(8 \mathrm{~h}$ light at $22^{\circ} \mathrm{C}$, and $16 \mathrm{~h}$ dark at $\left.18{ }^{\circ} \mathrm{C}\right)$, and vernalized at $4{ }^{\circ} \mathrm{C}$ in short days $(8 \mathrm{~h}$ light $/ 16 \mathrm{~h}$ dark). To compare the duration of flowering between the pep1, 35S:AaFLC dsRNAi line and wild-type plants, both genotypes were first grown for 5 weeks in long days (growth cabinet), vernalized for 12 weeks and then transferred back to long days. Flowering time was measured by scoring the leaves on the main shoot of at least eight individuals. Node position on the main shoot was determined by the presence of a true leaf and numbered from the bottom to the top of each plant.

Characterization of $\boldsymbol{p e p} 1$ mutant. Total RNA was extracted using an RNeasy Plant Mini Kit (Qiagen). Full-length AaFLC cDNA was amplified from expanded leaves and apices using primers on $5^{\prime}$ UTR (5'-AAACACAAAAAA AGAGTGAGAATAG-3') and 3' UTR (5'-AGTCTCTCAGCCATAGAGAG-3') (30 cycles). PCR products were cloned into a pCR2.1-TOPO vector and 14 clones were sequenced. Genomic DNA from pep1 mutant was extracted using the DNeasy DNA kit (Qiagen), and the AaFLC gene was sequenced using primers spanning the whole locus (primer sequences available on request). $F_{2}$ progenies were genotyped for the mutation in AaFLC using primers 5' -TTTGCCCCTTAG TTTTGTGG-3' and $5^{\prime}$-TACCCGGGAAGACTACATGC-3' flanking the mutation, and subsequently sequencing using primer $5^{\prime}$-TTTGCCCCTTAGTT TTGTGG-3'.

BAC analysis and sequencing. A BAC library was constructed using the pIndigoBAC-5 vector from partially HindIII-digested total genomic DNA of the accession Pajares (R. Castillo, unpublished data). The BAC library was screened using Arabidopsis FLC as a probe (without the MADS-box coding sequence) and two positive BACs were identified. Plasmid DNA was extracted from $100 \mathrm{ml}$ overnight culture using the Qiagen Plasmid Midi kit. Positively hybridizing BACs were analysed by fingerprinting and all corresponded to one locus. The DNA sequence of a positively hybridizing BAC was determined using the Sanger method ${ }^{31}$ and assembled using PHRED ${ }^{32,33}$, PHRAP and Consed ${ }^{34}$. Gene prediction from GenMark.hmm ${ }^{35}$, FGeneSH $^{36}$ and GenomeThreader ${ }^{37}$ using several plant expressed-sequence-tag databases were integrated manually using the APOLLO genome editor ${ }^{38}$. Manual functional annotation of all predicted genes was on the basis of their homology to A. thaliana and other plant species, as well as on the presence of conserved domains identified using the InterPro $^{39}$ database.

Plasmid constructs. To generate the 35S::AaFLC construct, the full-length (ATG to TAG) coding sequence of AaFLC was PCR-amplified with primer pair X-AaFLC5-F, 5' -(attB1)GAGACAGAAGCCATGGGTAG-3', and X-AaFLC3$\mathrm{R}, 5^{\prime}$-(attB2)GGCTTAATTGAGTAGTGGGAG-3' , and verified by sequencing after cloning into pDONR221 (Invitrogen). Through site-directed recombination it was subsequently cloned into a binary vector pLEELA, which is a derivative of pJawohl3 RNAi (GenBank accession AF404854) containing a GATEWAY cassette introduced into the HpaI site.

To make the AaFLC dsRNAi construct for transformation of A. alpina, a fragment including sequences from exon 4 to $3^{\prime}$ UTR was amplified from cDNA using primer pair AaFLC-RNAi-F1, 5'-(attB1)AAGCTCGTGGCA CCAAATGTC-3' ${ }^{\prime}$, and AaFLC-RNAi-R1, 5' ${ }^{\prime}$ (attB1)AGTCTCTCAGCCATAGA GAGT- $3^{\prime}$. The sequence of the AaFLC RNAi fragment is between coordinates 364 and 763 in the AaFLC/PEP1 cDNA sequence (GenBank Accession Number FJ755930). The fragment was recombined to generate a GATEWAY entry clone and subsequently recombined into the binary vector pJawohl8 (GenBank accession AF408413) to generate an intron-spliced hairpin construct.

Gene expression analysis. Total RNA was extracted from expanded leaves and shoot apices (approximately $1 \mathrm{~mm}$ in length), and an on-column DNase treatment (Qiagen) was performed to reduce any DNA contamination. RNA was analysed by qRT-PCR using a BioRad iQ5 apparatus and SYBR Green I detection. Three micrograms of total RNA was used for CDNA synthesis with oligodT15 as a primer. cDNA was diluted to a final volume of $180 \mu \mathrm{l}$, and $3.5 \mu \mathrm{l}$ of diluted cDNA was used for PCR. An A. alpina RAN3 gene was used as a control to normalize the amounts of cDNA. For testing AaFLC/PEP1 expression with qRTPCR, the primer pair PEP1-RT-F1, 5'-CTTGTCGTCTCCTCCTCTGG-3', and PEP1-RT-R1, 5'-ACTACGGCGAGAGCAGTTTC-3' were used. Primer pair AaRAN3-F2, 5'-CACAGGAAAAACCACATTCGT-3', and AaRAN3-R2, 5' CCATCCCTAAGACCACCAAAT-3' were used to detect AaRAN3 transcript abundance.

In situ hybridization. Methods of digoxigenin labelling of mRNA probes, tissue preparation and in situ hybridization were performed as already described ${ }^{30}$. Template DNA used for probe synthesis to detect AaFLC/PEP1 transcripts were
PCR-amplified from cDNA using the primer pair T3-PEP1-F2, 5'-ATTA ACCCTCACTAAAGGGAAGCCAGATGGAGAAGAAGAC-3', and T7- $\overline{P E P 1-}$ R2, 5'-TAATACGACTCACTATAGGGAACAAGGGTACGAAGATCCA-3'. The underlined nucleotide sequence indicates either the T3 or the T7 RNA polymerasebinding sites.

Chromatin immunoprecipitation. ChIP were carried out as described ${ }^{30}$. The antibody anti-H3K27me3 (Upstate) was used for the immunoprecipitation and an anti-rat IgG (Sigma) was used as a negative control. Two independent immunoprecipitations were performed with the anti-H3K27me3 antibody as technical replicates, and average values were obtained. The ChIP DNA was subjected to 33-34 cycles of PCR (non-saturated conditions). Primers used for PCR were designed to avoid the duplicated region in the AaFLClocus represented in Fig. 4h (Supplementary Table 2). RAN3 amplification was used to normalize DNA concentrations. The semi-quantification of the data was performed using the QIAxcel System (Qiagen) and the QX DNA size marker 50-800-bp (Qiagen). An appropriate dilution of the input amplified in similar conditions was used to compare the ChIP data. Results are presented as a ratio of vernalized/non-vernalized samples and are the means of two technical replicates. Similar patterns of modification were obtained in a second set of biological replicates.

Scanning electron microscopy. Plants of similar sizes were selected and marked on the main shoot just below node 15 (as in Supplementary Fig. 2). Apices from the main shoot were sampled to include leaves grown above node 15 at: 8 weeks in long days, after 12 weeks in vernalization and after 1 subsequent week in long days. Expanded leaves were removed from shoots apices and fixed in $2.5 \%$ glutaraldehyde in $0.002 \mathrm{M}$ phosphate buffer $(\mathrm{pH} 7)$ and $0.01 \%$ Nonidet P-40. Samples were dehydrated in ethanol and critical-point dried in liquid $\mathrm{CO}_{2}$. Leaf primordia were removed under a binocular microscope and samples were mounted on stubs, coated and subjected to high resolution Zeis Supra 55 VP FEG scanning electron microscopy with a Gatan Alto 2500 cryo system. The presence of flowers or flowering shoots and the identity of shoots in the axils of each leaf were recorded starting from the top of each sample until the lower leaf, which corresponded to the leaf in node 15. Each plant was scored at each developmental stage. At every node, the presence or absence of a shoot was recorded, shoots were excised and leaf primordia were removed under the binocular microscope and subjected to high resolution scanning electron microscopy.

Phylogenetic analysis. Protein-protein BLAST (http://www.ncbi.nlm.nih.gov/ BLAST) was performed using the deduced amino acid sequence from AaFLC. ClustalX was used to create multiple alignments ${ }^{40}$, and a phylogenetic tree was generated by the neighbour-joining method with Splitstree ${ }^{41}$. Bootstrap analysis was performed to estimate nodal support on the basis of 1,000 replicates. The accession numbers were: FLC, NP_196576; MAF1, NP_177833; MAF2, Q9FPN7; MAF3, NP_201311; MAF4, NP_201312; MAF5, AAO65320; AlFLC, AAV51231; ThFLC, AAY34137; AsFLC, AAZ92553; ArFLC, AAZ92552; BnFLC1, AAK70215; BnFLC2, AAK70216; BnFLC3, AAK70217; BnFLC4, AAK70218; BnFLC5, AAK70219; BrFLC1, ABI29999; BrFLC2, ABO40820; BrFLC3, ABI30001; BoFLC1, CAJ77613; BoFLC3, AAP31677; BoFLC4, AAQ76273; SaFLC, ABP96967; RsFLC, AAP31676; and AGL42, NP_568952.

31. Sanger, F., Nicklen, S. \& Coulson, A. R. DNA sequencing with chain-terminating inhibitors. Proc. Natl Acad. Sci. USA 74, 5463-5467 (1977).

32. Ewing, B., Hillier, L., Wendl, M. C. \& Green, P. Base-calling of automated sequencer traces using phred. I. Accuracy assessment. Genome Res. 8, 175-185 (1998).

33. Ewing, B. \& Green, P. Base-calling of automated sequencer traces using phred. II. Error probabilities. Genome Res. 8, 186-194 (1998).

34. Gordon, D., Abajian, C. \& Green, P. Consed: A graphical tool for sequence finishing. Genome Res. 8, 195-202 (1998).

35. Lukashin, A. V. \& Borodovsky, M. GeneMark.hmm: new solutions for gene finding Nucleic Acids Res. 26, 1107-1115 (1998).

36. Salamov, A. A. \& Solovyev, V. V. Ab initio gene finding in Drosophila genomic DNA Genome Res. 10, 516-522 (2000).

37. Gremme, G., Brendel, V., Sparks, M. E. \& Kurtz, S. Engineering a software tool for gene structure prediction in higher organisms. Inf. Softw. Technol. 47, 965-978 (2005).

38. Lewis, S. E. et al. Apollo: a sequence annotation editor. Genome Biol. 3, research0082 (2002).

39. Apweiler, R. et al. InterPro-an integrated documentation resource for protein families, domains and functional sites. Bioinformatics 16, 1145-1150 (2000).

40. Thompson, J. D., Gibson, T. J., Plewniak, F., Jeanmougin, F. \& Higgins, D. G. The CLUSTAL_X windows interface: flexible strategies for multiple sequence alignment aided by quality analysis tools. Nucleic Acids Res. 25, 4876-4882 (1997).

41. Huson, D. H. SplitsTree: analyzing and visualizing evolutionary data. Bioinformatics 14, 68-73 (1998). 